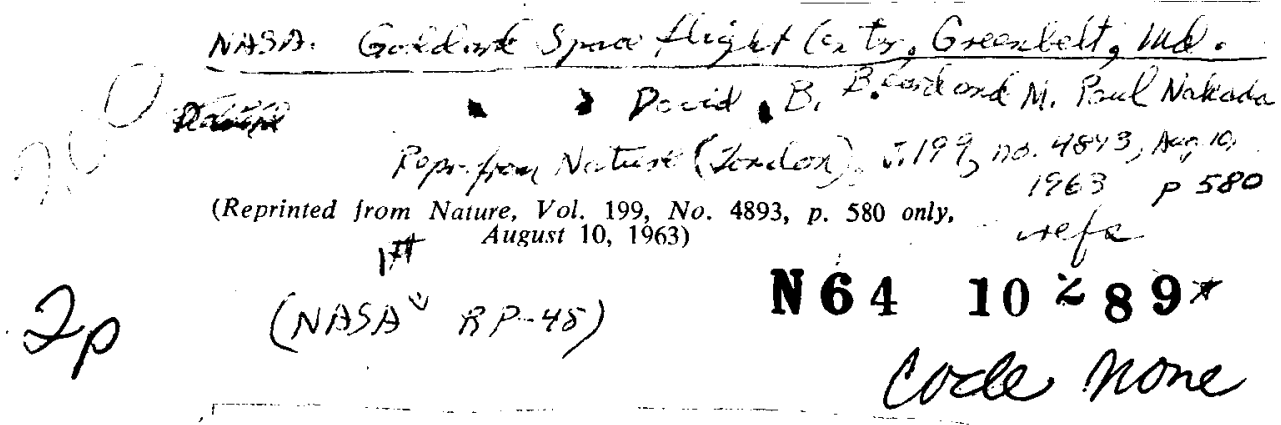

\title{
Interplanetary Magnetic Fields as a Cause of Comet Tails
}

IT has been generally recognized recently that the historic explanation of comet tails in terms of solar radiation pressure by either light or particles is impossible. Solar light pressure has been found to be insufficient by several orders of magnitude. Coulomb collisions or acceleration coupled with charge transfer by protons in the solar wind have also been shown by Bierulann and Treffitz ${ }^{1}$, in particular, to be grossly insufficient processes to account for comet tails streaming within a cylinder of small diameter and great length away from the Sun. It is the purpose of this communication to point out that the gases in cometary comas will be efficiently ionized by the solar wind of ionized hydrogen embedded in a magnetic field ${ }^{2-4}$ and that an interplanetary magnetic field will couple the cometary gas to the solar wind.

The neutral gases evaporated from comet nuclei will be ionized both by thermal electrons ${ }^{4}$ in the solar wind which have energies of several tens of electron volts and, as Heubner ${ }^{5}$ has emphasized, by Alfvén's ${ }^{6}$ process of ionization of a neutral gas by a fast moving plasma embedded in a magnetic field.

A comet head may be regarded as a densely ionized plasma essentially stationary in a high-pressure solar wind containing a low-pressure magnetic field moving with the wind. Recent satellite measurements ${ }^{4}$ have established that the solar wind consists of plasma the protons of which have an isotropic thermal energy of a few electron volts and a kinetic energy due to their stream velocity of a kilovolt or so. The interplanetary magnetic field observed to be embedded in the plasma is about $5 \boldsymbol{\gamma}$ ( $1 \gamma$ is $10^{-5}$ gauss). Thus the magnetic pressure, $H^{2} / 8 \pi$, is about equal to the thermal (isotropic) pressure of the wind in the moving co-ordinate frame of the wind.

The stream pressure of the solar wind, however, is the overwhelmingly dominant pressure in space, being two orders of magnitude larger than the free space magnetic pressure. Hence, the interplanetary magnetic field is compressed against any obstacle such as the stationary plasma provided by the comet coma or the stationary magnetic field provided by the geomagnetic dipole. Just as in the case of the example furnished by the geomagnetic dipole ${ }^{7}$, the interplanetary field is compressed against the obstacle offered by the comet coma so that the magnetic 
pressure increases to approximate equality with the stream pressure. If the interplanetary field is parallel to the solar wind velocity, the compressed field tails off parallel to the, solar wind in a cylindrical shape for a large distance downstream from the comet coma confining the comet plasma within this volume. If the interplanetary field is perpendicular to the wind velocity, the compressed field lines on

$\because$ the solar side of the comet slip around the edge of the comet and then continue moving with the solar wind?. The comet plasma will become ombedded in the moving field lines and will be carried radially away from the Sun, acquiring the velocity of the magnetic field as Heubners first proposed.

Quite simply the magnetic field may be regarded as the mechanism by which the solar wind is efficiently coupled to the comet plasma which Coulomb collisions are not able to provide. Fluctuations in solar wind pressure, or the magnetohydrodynamic waves the fluctuations generate, will also create the accelerations in comet tails which first led Biermann ${ }^{2}$ to deduce the presence of the solar wind.

\section{David B. Beard * \\ M. Paul Nakada}

Goddard Space Flight Center,

National Aeronautics and Space Administration, Greenbelt, Maryland.

* National Academy of Sciences-National Research Council Research Associate with N.A.S.A. Permanent address: Physies Department, University of California, Davis.

${ }^{1}$ Biermann, L., and Treffitz, E., Z. Astrophys., 49, 111 (1960).

${ }^{2}$ Biermann, I., Mém. Soc. Roy. Sci. Liège, Ser. 4, 13, 291 (1953).

3icrmam, L., Nuovo Cimento, Supp. 13,189 (1959).

4Neugebauer, M., and Snyder, C., Science, 138, 1095 (196\%). Coleman, jun., P. J., Davis, jun., L., Smith, E. J., and Sonnett, (., l', , Nience, 138, $1099(1962)$.

s Heubner, W. T., Revs. Mod. Phys., 33, 498 (1961).

- Alfvén, H., Rev. Mod. Phys., 32, 710 (1960).

- Beard, 1). B., J. Geophys. Res. (to be published). 